\title{
¿QUÉ OPINAN LAS PERSONAS SORDAS SOBRE EL APRENDIZAJE DE LA LENGUA ESCRITA?
}

\section{What do deaf people think about learning the written language?}

\section{Clara Elena Cruz Marte ${ }^{1}$}

Recibido: 14/5/2017 • Aprobado: 3/10/2017

DOI: http://dx.doi.org/10.22206/cys.2017.v42i4.pp73-82

\section{Resumen}

Cuando la mayoría de los docentes de personas sordas se refieren al desempeño de sus estudiantes en el uso de la lengua escrita suelen hacerlo con miradas, posturas y ademanes acompańados de palabras indulgentes que reflejan una realidad irresuelta y categórica: no son buenos lectores ni escritores, pero ¿opinan lo mismo las personas sordas?, ¿qué piensa esta comunidad al respecto? Con estas preguntas en el tintero iniciamos un estudio cualitativo de tipo exploratorio con tres propósitos en la mira: explicar la relevancia que le otorgan las personas sordas a la lengua escrita, conocer sus opiniones acerca de las posibilidades de desarrollar la competencia comunicativa en esta modalidad e identificar qué deben enseñar los docentes -desde la perspectiva de los encuestados- para formar estudiantes competentes en el uso de la lengua escrita. El muestreo está compuesto por 12 sujetos sordos adultos prelocutivos, usuarios de la lengua de señas. El rango etario de la población objeto de estudio es de 25 a 28 años, con excepción de dos personas que sobrepasan esa edad (42 y 47 años). La población consultada respondió una encuesta por escrito, cuyos resultados fueron validados en dos grupos focales. El análisis de las voces de las personas sordas consultadas corrobora que esta comunidad desea ser competente en el uso del español escrito porque considera que tiene la capacidad

1. Es candidata a doctora de Estudios del Español: Lingüística y Literatura de la Pontificia Universidad Católica Madre y Maestra (PUCMM), República Dominicana.

E-mail: claraelena27@gmail.com para desarrollar esa competencia, siempre y cuando los docentes hagan lo necesario para lograrlo. Quedó en evidencia el desafío que deben asumir las instituciones formadoras del país para lograr que los maestros de sordos sean bilingües, con dominio de variadas metodologías para desarrollar a su vez las competencias comunicativas de sus estudiantes, tanto en lengua de señas como en español. De igual forma, salió a la luz la necesidad de detectar oportunamente a toda la población que nace con pérdida de audición.

Palabras clave: competencia comunicativa; educación de sordos; lengua de seńas; enseńanza de la escritura; bilingüismo.

Abstract
When teachers of deaf people refer to the performance of
their students in the use of written language, they usually
do so with looks, postures and gestures accompanied by
indulgent words that reflect an unresolved and categori-
cal reality: Teachers think that deaf people are not good
readers or writers; however, do deaf people have the same
opinion about themselves? What do they think about it?
With these questions, we began a qualitative exploratory
study with three purposes in mind: to explain the rele-
vance that deaf people give to the written language, to
know their opinions about the possibilities of developing
communicative competence in this modality and to iden-
tify what teachers should teach -from the perspective of
deaf people- to train students to be proficient in the use
of written language. Sampling is composed of 12 pre-
locutive adult deaf people, sign language users. The age
range of the population under study is 25 to 28 years,


with the exception of two people who exceed this age ( 42 and 47 years). The population consulted responded to a written survey whose results were validated in two focus groups. The analysis of the voices of deaf people consulted corroborates that this community wants to be proficient in the use of written Spanish, and they have the capacity to develop that competence as long as teachers do what is necessary to achieve it. It was evident the challenge that educational authorities and training institutions have in the country to achieve bilingual teachers with mastery of various methodologies that allow them to develop in turn the communicative skills of their deaf students in sign language and written Spanish. Similarly, the need to detect early the entire population born with hearing loss came to light.

Keywords: communicative proficiency; deaf people; sign language; written Spanish; bilingualism.

\section{Introducción}

Para la mayoría de las personas sordas las barreras para el acceso a la competencia de la lengua escrita escapa a su conciencia, por lo que en este estudio nos proponemos extraer de su experiencia y de sus necesidades comunicativas tres cuestiones fundamentales: su opinión acerca de la importancia de la lengua escrita en sus vidas, la facilidad o dificultad para desarrollar niveles óptimos de competencia comunicativa en la lengua española escrita y, en tercer lugar, cómo lograrlo, es decir, qué tienen que hacer los maestros - desde su perspectiva- para que ellos puedan acceder a la cultura escrita como lo exige el mundo eminentemente textualizado de hoy.

Existe mucha información sobre las barreras que enfrentan las personas sordas para acceder a la escritura; sin embargo, pocas miradas se han dirigido hacia la percepción que tienen ellos mismos sobre su situación. Dentro de los escasos precedentes sobre el tema, encontramos la investigación de Gutiérrez (2011) en la que analiza la autopercepción de estudiantes sordos y oyentes. Sus hallazgos muestran que ambos tienen una percepción positiva sobre su propia eficacia escritora. Existe otro estudio sobre las representaciones sociales que construyen las personas sordas adultas sobre la lectura y la escritura en el que la autora desentrańa la relación de la comunidad sorda con la lengua escrita. Este estudio determinó que la población investigada percibe el aprendizaje de esta modalidad "[...] como una lengua difícil que cuesta comprender tanto en la etapa de aprendizaje escolar como en su uso en la vida cotidiana” (Yarza, 2014, p. 107). Yarza concluye en su investigación que el aprendizaje de la lengua escrita aparece matizado con sentimientos y actitudes negativas.

Resulta pertinente seguir avanzando por esta senda de investigación etnográfica que propone desempolvar la voz de la población sorda en la República Dominicana, que por años ha sido objeto de estudios de toda índole (psicolingüístico, sociolingüístico, antropológico, pedagógico, audiológico, médico); así como rescatar su rol de sujetos con voz y pensamiento propios.

La mayor parte de la comunidad oyente califica las producciones escritas de las personas sordas como textos ineficaces, agramaticales, sintácticamente desorganizados, cuya coherencia y cohesión revelan muy poco dominio de la escritura. Las investigaciones de Herrera, Puente, Alvarado y Ardilla (2007, p. 270) afirman que "los lectores sordos enfrentan grandes dificultades para alcanzar niveles funcionales de lectura", pero lo preocupante es advertir que con mucha frecuencia solemos atribuir esas deficiencias a la condición de sordera, es decir, como el estudiante no escucha, no puede adquirir y desarrollar la lengua mayoritaria y, por lo mismo, no podrá ser competente en su versión escrita. Esto reduce las expectativas y los esfuerzos de los docentes. No obstante, entendemos que esa realidad forma parte de un círculo vicioso que empieza con la convicción de los educadores acerca de las "limitadas" posibilidades de los sordos para acceder con éxito a la cultura escrita, permea la práctica de aula con esta convicción y concluye 
en el punto inicial como comprobación de la profecía autocumplida.

Pero, ¿la comunidad sorda opina lo mismo?, ¿cuál es su apreciación sobre su propio aprendizaje del español escrito?, ¿cuál es su opinión sobre la eficacia docente al enseñarles esta modalidad? El interés por llevar a cabo esta indagatoria nace de la inquietud acerca del perjuicio que representa el hecho de que tanto los docentes como los estudiantes sordos configuren sus expectativas muy por debajo de sus posibilidades reales.

\section{Fundamentación teórica}

El mayor reto al que se enfrenta un docente en una escuela para sordos es lograr que sus estudiantes accedan a la lectura y a la escritura. Esto lo he venido escuchando y viviendo a lo largo de tres décadas de ejercicio profesional como maestra de sordos. Al respecto, Claros-Kartchner (2017, p. 85) explica que:

"debemos reconocer que el desarrollo de la lectoescritura en los sordos no es simplemente la adquisición de la forma escrita de su primer idioma, como es el caso de los nińos oyentes, sino la adquisición de un segundo idioma, el cual tiene una forma escrita”.

Es por ello que hablamos de bilingüismo, enfoque necesario para el desarrollo de las destrezas lingüísticas y cognitivas de las personas sordas. Nos apegamos a la acepción de bilingüismo referida a la condición lingüística de los sordos que emplean la lengua de señas como su primer idioma (Rodríguez, Hernández y Castellanos, 2007, p. 4) $y$, en nuestro caso, el español en su modalidad escrita. Igualmente nos estaremos refiriendo a la lengua escrita como manifestación gráfica de la oral.

El diseño de competencia comunicativa que empleamos en este análisis es el adoptado en el Diseño Curricular del Nivel Primario en su segundo ciclo
(2014, p. 28), referido a "la capacidad para actuar de manera eficaz y autónoma en contextos diversos, movilizando de forma integrada conceptos, procedimientos, actitudes y valores" (2014. p. 28). Asumimos de igual forma el utilizado por Marta Marín (2004, p. 14), en el que incluye las competencias lingüística, discursiva, textual, pragmática y enciclopédica como componentes de la competencia comunicativa.

La gran mayoría de los niños sordos nace en hogares en los que ambos padres son oyentes, lo cual explica por qué gran parte de estos niños ingresa a la escuela sin aún haber adquirido ninguna lengua. Este estudio da fe de ello, pues de doce personas sordas que componen la muestra, solo una es hija de padres sordos. Es preciso puntualizar la diferencia entre adquirir una lengua naturalmente por la exposición a ella desde el nacimiento, y aprenderla en forma metódica. La adquisición de la lengua materna (primera lengua o L1) de los nińos oyentes es equiparable con la adquisición natural de la lengua de señas en los hijos de padres sordos usuarios de esta, situación que favorece el aprendizaje del español escrito, que para las personas sordas constituye una segunda lengua o L2. El énfasis no es ocioso, dado que muchos sordos adultos no conocen una lengua de signos formal, sino que manejan un sistema gestual creado en su núcleo familiar.

Los niños sordos de padres oyentes padecen la privación lingüística de la que debe ser su primera lengua por no tener el modelo en casa. Para atenuar esto tendrían que darse por lo menos cuatro condiciones: que la detección de la sordera ocurra desde el nacimiento, de manera que los padres sepan que tienen un hijo sordo lo más temprano posible; que reciban la orientación adecuada para superar el duelo de manera oportuna; que aprendan la lengua de seńas; $y$, finalmente, que se comuniquen de manera fluida con su hijo sordo. Sin embargo, la realidad es muy distinta. Todavía en la República Dominicana no se realiza el tamizaje auditivo obligatorio en los centros de salud materno-infantiles, 
por lo que los padres se dan cuenta de la sordera de su hijo sobre los dos años de edad, cuando notan que el lenguaje verbal no se ha producido. Al respecto apunta Claros-Kartchner (2017. p. 92) que el desarrollo de la lectoescritura en estos niños se verá afectado negativamente por no haber desarrollado un primer idioma que medie en el desarrollo del segundo.

Tabla 1. Configuración de la población estudiada

\begin{tabular}{lcc}
\hline \multicolumn{1}{c}{ Población por grado de sordera y cronología } & Cantidad & Porcentaje \\
\hline Sordos profundos prelocutivos & 10 & $83.33 \%$ \\
Sordo profundo post-locutivo & 1 & $8.33 \%$ \\
Hipoacúsica de moderada a severa & 1 & $8.33 \%$ \\
Total & 12 & $100 \%$ \\
\hline Nivel educativo de la población & & \\
\hline Educación superior & 5 & $42 \%$ \\
Educación secundaria & 4 & $33 \%$ \\
Educación técnica & 3 & $25 \%$ \\
Total & 12 & $100 \%$ \\
\hline
\end{tabular}

En este análisis empleamos el concepto de sordera prelocutiva manejado en la Guía sobre la discapacidad auditiva de la Federación de Padres y Amigos del Sordo de Castilla y León (s.f.), que describe como prelocutiva la sordera que aparece antes de los 3 años, cuando aún no se ha desarrollado el lenguaje. La sordera postlocutiva es la que se produce cuando ya el niño o la niña ha adquirido o está en proceso de adquisición de su lengua por vía auditiva, entre los tres y los cuatro años de edad, lo cual impacta significativamente en el desarrollo de la competencia comunicativa en la lengua escrita.

Lo que nos mueve a buscar la perspectiva de la comunidad sorda en esta cuestión es nuestra convicción en el principio del nibil de nobis, sine nobis (nada sobre nosotros sin nosotros) que aplicado a este tema en particular constituye un rechazo a la habitual costumbre de tomar decisiones para 'mejorar' (así, entre comillas) aquello que nos parece que anda mal, pero sin consultar a los implicados, sin conocer sus puntos de vista.

76 | Ciencia y Sociedad 2017; 42(4): 72-82 • Diálogos
Creemos que nuestros modelos mentales nos guían, es decir, "las imágenes, supuestos e historias que llevamos en la mente acerca de nosotros mismos, los demás, las intuiciones y todos los aspectos del mundo (...) operan como un cristal que filtra la visión del mundo y determina lo que vemos." (Valderrama, 2011, p. 67), de manera que las concepciones, los juicios y prejuicios que tengan las personas sordas con respecto a la lengua escrita determinará su actitud hacia su aprendizaje. Nos resulta de gran valor conocer su visión, la representación social que han ido construyendo producto de la experiencia como estudiantes y usuarios de la lengua escrita, gracias a las facilidades que nos brindan las redes sociales y las tecnologías de la información y la comunicación.

Nos guiarán en este recorrido estas cuatro preguntas:

1. ¿Qué importancia tiene la lengua escrita para las personas sordas prelocutivas usuarias de la lengua de señas?

2. ¿Qué opinan sobre sus posibilidades de desarrollar buenos niveles de competencia comunicativa en la lengua escrita? 
3. ¿Los docentes en la República Dominicana les enseñan bien a los sordos a comunicarse por escrito?

4. ¿Qué y cómo deberían enseñarles para que puedan comunicarse por escrito eficientemente?

\section{Aspectos metodológicos}

El estudio fue realizado con doce personas sordas de la ciudad de Santo Domingo, República Dominicana. Diez de ellas forman un grupo etario cuasi homogéneo, con edades comprendidas entre los 25 y los 28 años. Solo dos están fuera de ese promedio con edades de 42 y 47 años, respectivamente. La población encuestada está conformada por seis mujeres y seis hombres.

Con relación a los niveles de sordera y el tiempo de aparición de la misma, las características son las siguientes: todos los sujetos de la muestra son sordos prelocutivos o prelingüísticos; once nacieron sordos y uno de ellos perdió la audición antes de los dos años de edad, lo que lo ubica en esta categoría. Con respecto al grado de sordera, uno de los doce tiene niveles de pérdida auditiva de moderada a severa, lo que significa que su audición residual le permite acceder a algunos segmentos del habla, siempre y cuando lleve prótesis auditivas bien adaptadas a su tipo y localización de la pérdida y esté funcionando adecuadamente; sin embargo, esta audición no le resulta funcional para percibir y procesar la información lingüística por la vía auditiva y haber desarrollado lenguaje oral. Los demás sujetos de la muestra son sordos profundos, con niveles de sordera por encima de los $90 \mathrm{~dB}$. De las doce personas que conforman la muestra, una es hija de padres sordos, los demás son hijos de padres oyentes, como suele suceder en la mayoría de los casos. Todos son usuarios de la lengua de señas.

Todos concluyeron su educación básica en la misma escuela para sordos y su educación secundaria en distintas escuelas regulares. Cinco de los jóvenes han accedido a formación universitaria en carreras de arquitectura, contabilidad e informática (el 42\% de la muestra) y tres de ellos a formación técnica en el área de informática y diseño gráfico, lo cual representa el $25 \%$ de la muestra seleccionada. El $33 \%$ concluyó la educación secundaria.

Un aspecto relevante para la selección del universo es que, además de ser personas sordas prelocutivas, debían contar con niveles educativos suficientes para contestar un cuestionario por escrito y ser capaces de emitir un juicio propio acerca de los temas consultados. La muestra se conformó con los sujetos identificados con las características descritas. El número supuso también manejabilidad en el análisis de los datos, dado que nos permitió capturar, corroborar y analizar en un tiempo prudente. Se trata de una muestra típica homogénea, en tanto que sus integrantes responden al prototipo de personas sordas en los aspectos biológicos, sensoriales, académicos, lingüísticos y culturales. Los participantes accedieron libre y voluntariamente a colaborar con el estudio.

Para la recolección de datos empleamos dos técnicas: la encuesta escrita y el grupo focal. El cuestionario para la encuesta contiene preguntas de respuesta abierta y cuatro opciones de respuesta cerrada en cada pregunta, con la finalidad de sistematizarlas con mayor precisión. La captura de datos se realizó a través de medios impresos (papel y lápiz) y del sistema celular de mensajería instantánea WhatsApp. Elegimos esta técnica de cuestionario escrito por tres razones esenciales: la facilidad de aplicación -a pesar de las distancias- a través de medios electrónicos; la disponibilidad de tiempo para los encuestados, lo que les permitió procesar la información y responderla sin prisas; y el conocimiento previo del tema para su posterior discusión en los grupos focales. En la validación del cuestionario participaron cinco expertas en el tema a través de la aplicación Formularios de Google. 
Estos grupos focales fueron realizados con la intención de validar las informaciones recabadas en las encuestas, al tiempo de generar entre los participantes un clima de intercambio de ideas que nos permitiera extraer sus opiniones de manera confiable. El primer grupo de discusión se llevó a cabo el 5 de diciembre de 2016 a las 11:30 a. m., con la participación de las tres personas sordas de mayor edad (28, 42 y 47 años). El joven de 28 ańos comparte más características de afinidad con los mayores que con el grupo más joven. El segundo encuentro se realizó el 7 de septiembre de 2016 a las 11: $30 \mathrm{a}$. m. con los demás participantes -los del segmento etario de 25 a 27 ańos-. Con ese agrupamiento quisimos asegurar que la discusión discurriera en la confianza que generan las características generacionales.

En el primer grupo de discusión nos apoyó una experta de confianza para los encuestados. En el segundo, contamos con el soporte de una persona sorda con suficiente conocimiento del contenido del cuestionario y con buen manejo de la lengua de señas; esto con la finalidad de eliminar barreras en la comunicación y evitar confusiones. Ambos grupos focales fueron grabados en video con el conocimiento y la anuencia de cada uno de los participantes.

\section{Resultados}

A la primera pregunta: ¿qué importancia tiene la lengua escrita para las personas sordas prelocutivas usuarias de la lengua de señas? 11 personas de 12 (el 91.6\%) respondieron que para ellas la lengua escrita es necesaria para insertarse en el mundo laboral, para comunicarse con el mundo oyente-que es la gran mayoría, señaló uno de los participantes-; esto es, con el jefe, los docentes, la familia y con todo el que no sepa lengua de señas; para tomar los exámenes, resolver problemas; para aprender la gramática del español, para aprender más, porque es más fácil que la lengua oral, porque expresan sus deseos, porque hay que saber leer para desenvolverse en cualquier lugar -en el supermercado, en la farmacia, en la iglesia, etcétera-. Manifestaron que muchos oyentes no comprenden su expresión oral, por lo que recurren a la expresión escrita y a los gestos.

Como vemos, las personas sordas del estudio le otorgan importancia al uso de la lengua escrita en su vida. No la asumen como una imposición, sino como una necesidad para poder relacionarse con otros, para insertarse eficientemente en el mundo laboral y en la cotidianidad de un mundo textualizado y globalizado a través de los medios informáticos y de las redes sociales.

El señalamiento de que la lengua escrita es 'más fácil que la lengua oral' se refiere en este contexto a la dificultad que les representa la lectura labiofacial. En la oralidad las palabras son fugaces y en ocasiones confusas, por ejemplo, las que tienen fonemas con igual punto de articulación, como mapa y papa, o las que tienen fonemas guturales cuya articulación es invisible a la lectura de los labios. En este sentido, la escritura les otorga más tiempo para analizar, buscar significados y elaborar respuestas.

La encuestada que respondió que la lengua escrita "es más o menos importante" argumenta que para ella es muy difícil escribir sin una pauta que la guíe en la redacción, situación similar a la que ocurre con muchos estudiantes oyentes del nivel secundario (Gutiérrez, 2011; Cruz, 2008). De acuerdo con Díaz-Barriga y Hernández (2004), las causas que provocan que los estudiantes desarrollen escasas competencias de expresión escrita escapan muchas veces al propio sujeto; obedecen más bien a prácticas pedagógicas mal orientadas.

"Ha quedado demostrado que en las aulas a penas se destina tiempo para enseñar dichos procesos (leer y escribir) de forma explícita, desplegando prácticas ingenuas o estereotipadas que escasamente conducen a aprender 
dichos procesos como verdaderas actividades constructivas y estratégicas con sentido" (Díaz-Barriga y Hernández, 2004, p. 237).

Con respecto a las posibilidades de desarrollar buenos niveles de competencia comunicativa en la lengua escrita, diez personas sordas respondieron que sí, que pueden alcanzarlos; y dos contestaron que tal vez los pueden lograr. La relación es de un $76 \%$ contra un $24 \%$. Las razones que aducen quienes respondieron positivamente son que todos los sordos pueden hacerlo porque tienen la capacidad de aprender, que es más fácil que leer los labios porque [los oyentes] "mueven la cabeza y suelen olvidarse de hablar despacio y no siempre podemos prestar atención. A veces es agotador y a veces aburrido" (Valerio, comunicación personal, 17 de noviembre de 2016). Además, argumentaron que es una forma de expresar sus emociones y sentimientos. Las respuestas menos optimistas giran en torno al desconocimiento del vocabulario, al ingreso tardío a la escuela y a la desmotivación hacia la lectura y la escritura.

La República Dominicana todavía tiene irresuelta la detección oportuna de los nińos que nacen con pérdida auditiva, por lo cual ingresan tardíamente a la escuela. Así, el aprendizaje formal de la lengua de señas inicia cuando ya han pasado varios años sin recibir la estimulación lingüística necesaria para adquirirla y/o aprenderla. La comunidad sorda está inmersa en esta realidad; la limitación de las ofertas educativas inclusivas que respondan a sus necesidades lingüísticas y la escasez de centros educativos específicos que respondan a sus necesidades educativas son factores que agravan la situación. Con respecto a la desmotivación, sabemos que un individuo (sordo u oyente) que no haya aprendido ninguna lengua difícilmente pueda interesarse en la lectura o escritura de alguna.

Las respuestas a la pregunta ¿qué y cómo los docentes deberían enseñarles a los nińos sordos para que puedan comunicarse por escrito eficientemente? pusieron de manifiesto su reclamo de que se les enseñe en primer lugar la lengua de señas; en segundo lugar, el español escrito; $y$, finalmente, la comunicación oral. Resaltaron que los docentes deben asumir actitudes de paciencia, amor, esfuerzo y dedicación y hacer énfasis en la lengua escrita, cuya enseñanza encuentran poco eficiente. Transcribimos los comentarios de las personas sordas encuestadas por su relevancia en este estudio.

- Primero [deben enseñarnos] la lengua de señas y después la lengua escrita.

- A los niños sordos hay que enseñarles las palabras una por una por escrito, con señas y expresiones para que comprendan más rápido.

- Primero lentamente. En el nivel inicial [los maestros deben] enseñar la palabra en lengua de señas, y segundo, tienen que enseñar la palabra con su escritura.

- Primero deben enseñar a los niños a leer y a comprender y luego enseñarles a escribir el español con su gramática.

- Los niños sordos deben aprender a leer desde una temprana edad. Cuando yo era pequeño, mis padres me enseñaron a leer revistas y cuentos. $M i$ mamá me compró tres libros para aprender a escribir oraciones, verbos, etc. También me leia un párrafo y luego me explicaba de qué se trataba. Me corregian cuando me equivocaba.

- [Los maestros tienen que enseñar] con amor, paciencia, esfuerzo, siendo positivos, leyendo todos los dias cualquier libro, ayudándolos a aprender a comunicarse por escrito.

- Prestándoles más atención. Dedicándoles más tiempo.

- Por medio de actividades de lectoescritura.

Las referencias que hicieron los sujetos encuestados a la importancia y la necesidad de aprender la gramática del español nos parecen de gran significación, 
porque justamente este ha sido el talón de Aquiles en la historia de la educación para las personas sordas: la dificultad para aprender la gramática de la lengua de la comunidad predominante -el español, en nuestro caso-. Esta dificultad ha sido adjudicada a los sordos como una consecuencia propia de su condición, con toda la autoridad que otorga la posición docente; sin embargo, en este estudio notamos que el $16.6 \%$ de los participantes reclama que los docentes deben responsabilizarse de la enseñanza de la gramática del español en lugar de escudarse con excusas.

Expresarnos por escrito en español o en cualquier lengua con un sistema de escritura requiere, además del conocimiento léxico semántico y pragmático, el manejo de su morfosintaxis o estructura gramatical, de lo contrario, la producción escrita en esa lengua será deficiente. A esto se añade el hecho de que la gramática de la lengua de señas es distinta a la del español (Aldrete, 2009), como lo son las gramáticas del inglés y el español, por ejemplo. De manera que, si queremos estudiantes que escriban en español, tenemos que enseñarles su gramática y no solo el léxico. Entonces, ¿a quién le corresponde conocer las interioridades y pormenores de ambas lenguas?, ¿quién es el responsable de enseñarla? La obviedad de la respuesta salta a la vista.

Uno de los encuestados refirió que existen docentes que emplean un modelo muy simplificado de lengua escrita y uno muy mal estructurado de lengua oral para comunicarse con los sordos, con la ingenua intención de mejorar la comunicación. Esta práctica, lejos de ayudar, va en su detrimento, dado que les proporciona un patrón lingüístico erróneo (Guzmán, comunicación personal, 5 de diciembre de 2016). Esta aseveración debe quedar como una apelación de parte de la comunidad sorda a la conciencia y al deber de los docentes.

Para investigar la opinión de los encuestados acerca de los docentes de educación para sordos en la República Dominicana, preguntamos: ¡crees que en las escuelas de la República Dominicana los profesores enseñan bien a los sordos a comunicarse por escrito? El 50\% contestó que no, el 33\% respondió que tal vez y el $17 \%$ no contestó. El $50 \%$ que respondió negativamente argumentó que a los docentes les falta capacidad, conocimiento o capacitación para enseñarles el español escrito; que se comunican muy poco en esta modalidad porque basan sus clases fundamentalmente en las señas, que pierden el tiempo, que les falta paciencia y enseñarles más a los padres. El 33\% que respondió que tal vez alegó que los profesores que enseñan bien son aquellos que utilizan los métodos adecuados y enseñan con amor.

De las respuestas anteriores colegimos que las personas sordas están muy conscientes de que, para poder desarrollar competencias comunicativas en la lengua escrita, se necesitan docentes con el conocimiento metodológico necesario para poder enseñarla de manera eficiente, con actitud positiva y comprometida, que crean en sus capacidades y usen con frecuencia la modalidad de la lengua que enseñan con fines comunicativos.

\section{Conclusiones}

El análisis de las voces de las personas sordas consultadas nos permite corroborar que esta comunidad desea ser competente en el uso del español escrito y se siente en capacidad de ello; por tanto, no existe indisposición ni rechazo al aprendizaje de este. El desarrollo de estas competencias se alcanza cuando los docentes hacen lo necesario para enseñarles el español escrito con conocimiento de metodologías apropiadas y motivadoras, con paciencia, mediante el uso de la lengua escrita en situaciones funcionales, sin excesiva simplificación ni alteración de la morfosintaxis. Para ello, los docentes deben enseñarles la lengua de señas y desarrollar el lenguaje en esta primera lengua como condición necesaria para poder acceder al español como segunda lengua. 
Por otra parte, es imperioso que en la República Dominicana se establezca oficialmente, como parte de las evaluaciones rutinarias, el tamizaje auditivo en todos los centros de salud materno-infantil, sobre todo en los recién nacidos cuyas madres han cursado un embarazo de alto riesgo, como forma de agilizar el acceso oportuno de los padres y del niño a la lengua de señas como sistema de comunicación que garantice el desarrollo lingüístico en una primera lengua.

Ratificamos la necesidad de que los docentes manejen con suficiente fluidez y pericia las lenguas que enseñan, esto es, la lengua de señas como primera lengua o L1 y el español como segunda lengua o L2. El conocimiento y la fluencia de los docentes en la lengua de señas le permite al sujeto sordo desarrollar el lenguaje y con él las destrezas cognitivas necesarias para aprender cualquier otra lengua. Igual situación ocurre con el español escrito. En definitiva, esto constituye todo un desafío para las autoridades educativas del país, así como para los lingüistas y estudiosos del tema en cuestión.

\section{Referencias}

Aldrete, M. (2008). Gramática de la lengua de señas mexicana. Estudios de lingüística del español. (Tesis doctoral). El Colegio de México, México.

Alegría, J. y Domínguez, A. (2009). Los alumnos sordos y la lengua escrita. Revista latinoamericana de educación inclusiva, 3(1), 95-111. Recuperado el 20 de junio de 2017 de http://www.rinace.net/rlei/ numeros/vol3-num1/art7.pdf.

Claros-Kartchner, R. (2009). La inclusión de las personas sordas, como grupo étnico, en los sistemas educativos. Revista Latinoamericana de Educación Inclusiva, 3(1), 63-75. Recuperado el 20 de junio de 2017 de http://www.rinace.net/rlei/numeros/ vol3-num1/art5.pdf.
Claros-Kartchner, R. (2017). La desmitificación de la educación de sordos: hacia una pedagogía de éxito. Santo Domingo: el autor.

Cruz, M. (2011). Reflexiones sobre la Educación Bilingüe Intercultural para el sordo en México. Revista Latinoamericana de Educación Inclusiva, 3(1), 133-145. Recuperado el 20 de junio de 2017 de http://www.rinace.net/rlei/numeros/vol3num $1 /$ art $9 . p d f$

Cruz, C. (2008). Estrategias para mejorar la producción escrita en el nivel medio desde un enfoque metacognitivo. (Tesis de maestría). Universidad de Alcalá-Universidad APEC, Madrid-Santo Domingo.

Díaz-Barriga, F. y Hernández, G. (2004). Estrategias docentes para un aprendizaje significativo: una interpretación constructivista. México: MacGraw-Hill.

Federación de Padres y Amigos del Sordo de Castilla y León (FiapasCyL) (s/f). Guía sobre la discapacidad auditiva. Recuperado de http://www. saludcastillayleon.es/sanidad/cm/gallery/Aula $\% 20$ de\%20Pacientes/Gu\%C3\%ADa\%20sobre\%20 Discapacidad\%20Auditiva.pdf

Fernández, J. (2003). Creencias y modelos mentales en los perfiles competenciales. Recuperado de http:// www.degerencia.com/articulo/creencias_y_modelos_mentales_en_los_perfiles_competenciales

Gutiérrez, R. (2011). Auto-percepción de la eficacia en la escritura de alumnos sordos y oyentes de Educación Primaria y Secundaria. Electronic Journal of Research in Educational Psychology, 9(25), 1353-1376.

Herrera, V., Puente, A., Alvarado, J. y Ardilla, A. (2007). Códigos de lectura en sordos: la dactilología y otras estrategias visuales y kinestésicas. Revista Latinoamericana de Psicología, 39(2), 269-286. Recuperado el 15 de agosto de 2017 de http://www. institutodelasordera.cl/v2/wp-content/uploads/ codigos-lectura-sordos-dactilologia-otras-estrategias-visuales-kinestesicas.pdf

Ciencia y Sociedad 2017; 42(4): 72-82 • Diálogos 
Hinojosa, M. (2016). Procesos de adquisición de la lengua escrita en población infantil con pérdida auditiva. (Disertación doctoral, Universidad Complutense de Madrid). Recuperado de http:// www.rinace.net/rlei/numeros/vol3-num1/art9.pdf

Mata, F. y Cáceres, R. (2006). El proceso de planificación en la expresión escrita de alumnos sordos: estudio de casos en Educación Secundaria. Revista de educación, (339), 435-453.

Marín, M. (2004). Lingüistica y enseñanza de la lengua. Buenos Aires: Aiqué.

Ministerio de Educación de la República Dominicana. (2014). Diseño Curricular Primario. Segundo Ciclo, 4to, 5to y 6to. Santo Domingo: MINERD. Recuperado de http://www.ibe.unesco.org/curricula/ dominicanrepublic/dr_upr_2014_spa.pdf

Rodríguez, X., Hernández, I. y Castellanos, R. (2007). Modelo cubano de educación bilingüe para personas sordas: fundamentos y actualidad en el contexto nacional e internacional. La Habana: Órgano Editor Educación Cubana.

Rojas, A. (2015). Aportes de la investigación en el proceso lector en población sorda usuaria de la lengua de señas. Revista Areté, 15(2), 48-57. Recuperado en diciembre de 2015 de http://revistas.iberoamericana.edu.co/index.php/arete/article/ view/1014

Torres, M. (2009). El proceso de la escritura en estudiantes adolescentes sordos. Revista Latinoamericana de Educación Inclusiva, 3(1), 113131. Recuperado el 20 de junio de 2017 de http:// www.rinace.net/rlei/numeros/vol3-num1/art8.pdf Valderrama, B. (2011) Las bases psicológicas del coaching y el mentoring. Coaching y Psicologia, (251), 63-70.
Velasco, C. y Pérez, I. (2009). Sistemas y recursos de apoyo a la comunicación y al lenguaje de los alumnos sordos. Revista Latinoamericana de Educación Inclusiva, 3(1), 77-93. Recuperado el 20 de junio de 2017 de http://www.rinace.net/rlei/ numeros/vol3-num1/art6.pdf.

Yarza, M. (2014). Comunidad sorda y lengua escrita: un estudio acerca de las representaciones sociales que construyen las personas sordas adultas sobre la lengua escrita. (Tesis de maestría, Universidad Nacional de Quilmes). Bernal, Argentina. Recuperado de https://ridaa.unq.edu.ar/bitstream/handle/20. 500.11807/165/TM_2015_yarza_002.pdf?se quence $=1$

\section{Datos de filiación}

Clara Elena Cruz Marte. Es candidata a doctora de Estudios del Español: Lingüística y Literatura de la Pontificia Universidad Católica Madre y Maestra (PUCMM). Licenciada en Educación mención Filosofía y Letras por la UASD. Especialista en Lingüística Aplicada a la Adquisición de una Primera Lengua por el Instituto Mexicano de la Audición y el Lenguaje (IMAL, México). Magíster en Enseñanza del Español como Lengua Materna y como Lengua Extranjera por la Universidad de Alcalá (España). Se ha desempeñado como docente en diferentes universidades dominicanas, y es coautora de varias publicaciones del Ministerio de Educación de la República Dominicana.

Sus líneas de investigación versan sobre el acceso de las personas sordas al español escrito; metodologías para la enseńanza de su estructura morfosintáctica; producción literaria de y para personas sordas; lingüística aplicada al estudio de las lenguas de señas. Correo electrónico: claraelena27@gmail.com 